\title{
Live Streaming Commerce: A Review and Prospects
}

\author{
Yaoyuan $\mathrm{Fu}^{1, \mathrm{a}}$ \\ ${ }^{1}$ School of Business Administration, Chongqing Technology and Business University, Nanan District, \\ Chongqing, China \\ a1146986327@qq.com
}

\begin{abstract}
2016 has been called "the first year of live streaming" in China, and since then the live streaming industry has seen a blowout growth. The mode of "webcast + e-commerce" is a breakthrough to the traditional e-commerce model, and it is also an important monetization path for webcast. Live streaming commerce is all the rage in China, and an epidemic has pushed it even further. Theoretical circles are paying more and more attention to the emerging marketing model of live streaming. This study systematically sorts out the frontier research related to live streaming commerce, and carries out a comprehensive discussion on the definition, characteristics, types, mechanism of action and influence of live streaming commerce, and puts forward the practical enlightenment and future research direction. This research provides a meaningful guide for e-commerce companies to rationally use live marketing to improve consumer experience and purchase intentions.
\end{abstract}

Keywords: e-commerce, live streaming commerce, network celebrity

\section{直播带货研究综述及展望}

\author{
符铫袁 $1, \mathrm{a}$
}

${ }^{1}$ 重庆工商大学工商管理学院, 南岸区, 重庆, 中国

a1146986327@qq.com

\section{摘要}

2016 年被称为中国的 “直播元年” ，之后直播行业呈现井喷式的增长。“网络直播+电商” 的直播带货模式是 对传统电商模式的突破，也是网络直播重要的变现路径。直播带货风靡中国，一场疫情更是将其推向了热潮。 理论界对直播带货这一新兴营销模式的关注正逐渐升温。本研究系统地对直播带货相关的前沿研究进行了梳 理，对直播带货的定义、特点、类型、作用机制以及影响进行了整体性的探讨，最后提出了直播带货提高消费 者购买意愿的营销启示以及未来研究方向。本研究为电商企业合理运用直播营销提升消费者体验和购买意愿提 供了有意义的指南。

关键词: 电子商务, 直播电商, 网红

\section{1. 前言}

随着手机视频直播的出现, 网络直播 (1ive streaming) 成为年轻人休闲时的热门话题。2016 年 被称为 “中国网络直播元年”, 新冠疫情影响下, 直 播普及率进一步提高, 截至 2020 年 3 月, 直播用户 突破 5 亿。直播模式在中国的用途非常广泛, 如泛娱 乐 (即所谓的唱歌、跳舞、乐器、脱口秀主持人主持 和表演的 “展厅表演” ）、电子商务、个人知识分享、 个人经验分享、教育、以及医疗等 ${ }^{[1]}$ 。其中最火的莫
过于直播带货, 也叫做直播电商 (1ive streaming commerce) 这一新兴模式。主播 (e-commerce anchor) 通过网络平台直播发布产品或服务信息, 实时地运用 语言、形象、试用感受等形式向消费者宣传、推广产 品或服务, 吸引消费者做出购买决策 ${ }^{[2]}$ 。2020 年 7 月 6 日，人社部联合国家市场监管总局、国家统计局发 布 9 个新职业，带货主播成为了一种正式职业，被命 名为直播销售员，即在数字化信息平台上，运用网络 的交互性与传播公信力, 对企业产品进行营销推广的 人员。截至 2020 年底, 中国直播电商市场规模超 1.2 
万亿元, 年增长率为 $197.0 \%$, 行业内主播的从业人 数已经达到 123.4 万人 ${ }^{[3]}$ 。现在不仅网红 (network celebrity) 直播带货，明星、企业家、央视等主流媒 体甚至政府官员都纷纷涌入直播间卖货，非常热闹。 中国进入了 “万物可直播、人人能带货” 的时代。

为什么直播带货如此火爆, 与传统网上购物有何 区别？如何利用直播带货提高消费者购买意愿？其 背后的作用机制又是什么? 由于直播带货是新兴模 式, 目前学术界对这方面进行系统梳理的分析甚少, 因此当前急需对现有文献的脉络和结论进行整理和 分析, 为未来相关理论的开发和完善奠定基础; 从实 践来看, 研究成果的梳理能帮助商家更好地运用直播 带货这一模式推广产品和品牌。本文将基于营销视 角, 整理出直播带货的定义、特点、类型、作用机制 及影响, 提出通过直播带货增强消费者购买意愿的实 践建议, 并对未来的研究进行展望。

\section{2. 直播带货的定义、类型和特点}

\section{1. 直播带货的定义}

要理解什么是直播带货, 首先要理解什么是网络 直播。国家网信办对网络直播的定义是: 基于互联网, 以视频、音频、图文等形式向公众持续发布实时信息 的活动。随着直播越来越受欢迎, 社交电商平台上的 许多商家都将直播作为提高销售业绩的一种工具。这 导致了一种新的社交电商形式———直播电商的出 现, 俗称直播带货 ${ }^{[4]}$ 。

不同学者对直播带货给出了不同定义。裴学亮与 邓辉梅认为, 直播电商是指电子商务平台上的企业 (主要是零售商) 通过网络直播技术与顾客互动、宣 传、展示、体验商品, 并最终实现商品交易的线上零 售商业模式 ${ }^{[5]}$ 。魏华和高劲松等认为直播带货是主播 借助互联网平台, 以双向实时互动及场景化方式向用 户展示产品信息, 吸引用户积极参与的一种新型电子 商务营销模式 ${ }^{[6]}$ 。Jie Cai 和 Donghee Yvette Wohn 等认为, 从社交电商角度看, 直播电商平台本质上是 一个电子互动平台, 由大量的社交电商和特殊的媒体 属性组成。社交电商 (social commerce) 是电子商务 ( $e^{-}$-commerce) 的一个子类, 它利用支持互动性的社交 媒体来促进网上交易, 丰富消费者的网上购物体验 [7]。随着社交媒体的日益流行, 拥有众多粉丝的网红 产生了广告效果和销量。这些网红是可以在社交媒体 平台上影响他人的内容创造者 ${ }^{[8]}$ 。商家看中网红主播 的显著影响力, 雇佣他们直播。网红凭借自身的体验 和专业的分析, 以展示商品的功能和讲解为吸睛点, 激发用户的消费欲望, 从而带动产品销量 ${ }^{[9]}$ 。随着直 播带货的火热, 带货主播由网红扩展到了明星、企业 家、主流媒体甚至地方官员。

根据对相关概念的分析, 从营销的角度出发, 本 文对直播带货给出如下定义：直播带货是 “人、货、 场” 的重构, 是电商平台企业通过直播动态地介绍、
展示、推荐商品, 以实时互动的方式为消费者营造虚 拟的线上购物情景，从而激发消费者购买欲望和提高 消费者购物体验的营销模式。

\section{2. 直播带货的类型}

从平台来看, 可以分成两种情况: 一是电子商务 交易平台开通直播功能 ${ }^{[7]}$, 也就是电商直播化, 如京 东、淘宝。创业者、品牌方与商家开设直播间, 对自 家商品进行推广 ${ }^{[10]}$ 。二是可直播的流量平台嵌入电子 商务, 如抖音、快手、微博、小红书和Bilibili 等, 通过商品链接导向第三方交易平台或自建平台 ${ }^{[11]}$ 。

以主播划分, 可分为四类: 一是商家自播, 以购 物平台为主, 主播多为店铺或品牌商自有员工, 优势 是成本低, 直播场次多, 劣势是流量窄, 专业度不高; 二是达人主播, 李佳琪、微娅等职业网红主播或拥有 众多粉丝的明星在直播间汇聚售卖各种产品, 通过专 业知识或自身影响力带动粉丝, 为其推荐商品, 优劣 势与商家自播基本相反; 三是地方官员或主流媒体, 他们带货往往是销售当地农产品, 助力乡村振兴; 四 是虚拟主播, 如洛天依、乐正绫、初音未来等虚拟偶 像, 他们主要是吸引喜爱 “二次元” 的用户、突破用 户圈层限制 ${ }^{[3,10,11]}$ 。

\section{3. 直播带货的特点}

直播的盛行使图文货架式电商向直播电商转型, 这对传统商业是一次脱胎换骨的改造 ${ }^{[12]}$ 。相比传统商 业, 直播带货具有以下特点:

真实感和临场感: 在传统的网上购物中, 顾客只 能通过图片和文字了解产品。相比之下, 直播能让主 播全方位地展示产品特点和用途, 从而为客户提供更 详细的产品信息, 让消费者能够真实地感知商品, 体 验 “眼见为实” , 营造了一种面对面购物的情景, 增 强了消费者临场感 ${ }^{[13,14]}$ 。

实时交互性: 传统电视购物, 主播同样能通过现 场试用营造一种真实感, 但消费者困惑时无法提问, 对方也不能了解消费者的想法。直播带货情境下, 消 费者可以通过弹幕实时与主播交流，有效获取购物所 需信息。实时交互性有利提高用户观看体验和主播认 同, 进而影响消费者的满意度和行为 ${ }^{[15,16]}$ 。

传播广泛性: 截至 2020 年 12 月, 我国网民规模 达 9.44 亿, 短视频的用户使用率最高, 为 $88.3 \%$, 用户规模达 8.73 亿, 网络直播的使用率为 $62.4 \%$,

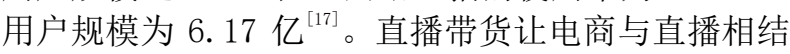
合, 使得营销内容以短视频的形式储存, 在庞大的短 视频用户助推下容易实现更广泛的传播 ${ }^{[15]}$ 。

\section{3. 直播带货的作用机制}

直播带货已经成为非常流行的营销模式，那么它 究竟是如何影响消费者购买意愿的呢? 本部分将主 
要基于感知价值理论、SOR 理论、匹配假说、信源可 信度和吸引力模式对直播带货影响消费者行为的原 因进行探讨。

\section{1. SOR 理论视角}

SOR (stimulus-organism-response)，即 “刺 激--机体--反应” 模型, 是由 Mehrabian 和 Russe1 于 1974 年提出, 被广泛应用于个体行为决策研究当 中。该理论表明外界刺激 (stimulus) 是个体产生心 理和认知 ( Organism) 变化的前提, 其变化会进一 步导致个体的行为（response）。

在直播带货下刺激因素 (stimulus) 主要包括信 息交互（个性化、响应性、娱乐性、互助性） ${ }^{[6]}$ 、网 络直播购物特征（互动性、真实性、可视性等) ${ }^{[14,18]}$ 、 互动 ${ }^{[19]}$ 、电商主播特征 (知名度、魅力性、推荐性、 展示性等) ${ }^{[20-22]}$ 、价格折扣、商家服务、增值内容 ${ }^{[23]}$ 以及社会临场感 ${ }^{[24]}$ 等。这些外在刺激通过关系强度、 兴奋感、感知价值（功能、享乐、情感价值等）、信 任感、心流体验、感知风险、感知愉悦、感知唤醒 ${ }^{[6,}$ 14, 18-24]等来影响消费者的行为。

\section{2. 感知价值理论视角}

感知价值是消费者基于成本效益权衡而感知到 的产品或服务的整体效用价值。一项技术或服务的成 功和采用是基于消费者从一项服务中感知到的或期 望得到的特定价值。

感知价值在维持与顾客的长期关系中起着重要 作用, 同时在情感上影响顾客的购买意愿。感知价值 被认为是意愿、持续意愿和忠诚的一个永恒的贡献因 素。Sonali Singh 等 (2021) 基于感知价值理论, 考察了影响用户使用网络直播服务的持续意图的各 种因素。研究结果表明, 便利性价值对用户继续使用 网络直播服务的意愿影响最大 ${ }^{[25]}$ 。Hongchang Mei 等 （2018）运用感知价值原则, 篎选出了直播背景下影 响消费者行为的因素。研究得出, 消费者的感知娱乐 性、社会性和有用性都对消费者的购买决策有显著的 正向影响。消费者感知风险对消费者购买决策具有显 著的负向影响 ${ }^{[26]}$ 。刘佳等 (2021) 研究得出消费者的 感知服务质量对购买意愿有正向影响, 并且感知价值 在其中起了中介作用 ${ }^{[27]}$ 。

\section{3. 匹配假说}

名人与产品的匹配: 代言的有效性取决于代言人 与品牌的匹配。研究人员已经探索了代言人和产品匹 配的重要性 ${ }^{[28,29]}$ 。大多数配对假说的研究都预测了代 言人一产品配对对产品态度、广告态度、购买意愿等 广告效果的各种衡量指标的影响 ${ }^{[30]}$ 。

自我协调理论: 人们选择购买和使用与自己的自 我形象一致的用户形象的商品和服务。消费者通过对 被认为与自我概念相似的品牌持有积极的态度和购
买来实现自我一致性 ${ }^{[31]}$ 。自我一致性解释并预测了消 费者行为的许多方面, 包括品牌态度、品牌偏好、 顾客满意度和购买意愿 ${ }^{[31,32]}$ 。这一观点表明, 自我与 产品的匹配程度越高, 购买意愿的可能性就越高。

Park 等（2020）基于名人代言和匹配假说的相 关研究, 以名人与产品、直播内容与产品、自我与产 品的一致性为研究对象, 探讨消费者如何看待网红与 直播内容, 以及影响购买意愿的因素 ${ }^{[8]}$ 。孟陆等将直 播网红特性与直播内容一致性定义为直播网红特性 与其在直播过程中创造内容之间的匹配程度。直播网 红特性与内容的一致性越高, 消费者认同感越强, 反 之则越弱 ${ }^{[9]}$ 。

\section{4. 信息源特性理论}

社会心理学中的信息源信度、效价模型认为, 信 息源可以从可信性、专业性和吸引力三方面特性影响 其对受众的说服效果, 并通过心理内化、心理顺从和 心理认同作用于个体的态度。高可信度的名人可以产 生有效的品牌信誉态度和购买意愿 ${ }^{[33]}$ 。

刘风军等 (2020) 认为, 针对网红信息源的特殊 性, 除包含上述 3 种特性外, 还应包含互动性。可信 性、专业性、互动性和吸引力四大特性共同组成, 相 互独立并能共同作用于受众内心感受, 增强对网红产 品的关注, 提升购买意愿。孟陆等 (2020) 将网红的 信息源特性划分为可信性、专业性、技能性、互动性 和吸引力五个维度, 探讨了不同类型直播网红对消费 者购买意愿的影响及其内在机制。

除了上述理论外, 还有学者从详尽可能性模型和 多重态度理论、替代学习视角 ${ }^{[35]} 、 \mathrm{TAM}^{[7]}$ 等理论研究 直播带货情境下的消费者行为。总体来看, 目前运用 最广泛的是 SOR 理论和感知价值理论。

\section{4. 直播带货的影响}

\section{1. 正面影响}

\section{1.1 产品或品牌层面}

在营销中，直播带货的互动性能够提升消费者与 品牌间的沟通质量，帮助品牌和产品获得消费者的正 面行为回应。网红借助直播、讨论组等新媒体通道与 消费者互动, 具有很强的实时动态双向互动性, 更能 唤起消费者的参与欲望, 这是传统名人代言广告无法 达到的效果 ${ }^{[9]}$ 。以头部网红主播李佳琦、薇娅直播为 代表, 通过叙说自己的经验故事或平台测评结果, 用 一种理性的方式向消费者分析品牌或产品的利弊, 广 受消费者的青睐，所卖商品短时间内便被一扫而空 [36]。

另外, 网红直播带货相比名人代言更容易让消费 者产生认同感。传统代言人多是当红明星, 依靠明星 光环来吸引消费者眼球, 更多依赖形象和知名度, 与 
消费者保持明星与偶像的关系, 是一种纯商业性质的 活动 ${ }^{[37]}$ 。而网红多是 “草根” 出身, 形象和知名度远 不及当红明星。其传播贴近大众生活的内容, 消费者 对于网红群体有更近的心理距离, 更容易与网红建立 起心理联结并产生认同感 ${ }^{[38]}$ 。

\section{1.2 公益层面}

直播带货在助力农产品销售方面也功不可没。直 播售卖农产品让消费者更加直观地看到农产品的生 产环节及存放的环境等, 缓解了农产品交易中的信息 不对称难题，使消费者买得明白、吃得放心。直播带 货农产品作为电商扶贫的一种新模式, 在帮助农民增 收、助力贫困户脱贫方面取得了显著的经济效益和社 会效益 ${ }^{[39]}$ 。

\section{2. 负面影响}

\section{2.1 消费者层面}

这几年直播电商行业的 “野蛮生长” 和无序竞 争, 也使得行业乱象频出。由于准入门槛低, 主播质 量良莠不齐, 损害消费者利益的事时有发生, 如货不 对板、重度观看成瘾、冲动性购买、夸大宣传、部分 商品掺杂掺假等问题, 加上监管不严和相关法律不健 全, 造成消费者维权困难 ${ }^{[2,14,25,40]}$ 。例如, 2020 年 11 月的网红主播辛巴燕窝售假事件。辛巴把风味饮 料夸大宣传为燕窝误导消费者购买。这件事的背后也 暴露出燕窝制品方面国家标准的缺失。

\section{2 .2 企业层面}

商家盲目相信 “直播带货神话” , 经营方面本末 倒置。部分厂商、商家期望通过邀请知名主播带货这 一营销方式, 快速地获得更高的经济回报, 却忽视了 基础性要素一一产品质量、服务品质 ${ }^{[41]}$ 。事实上, 商 家想通过网络直播冲销量, 一是需要与自带巨额流量 的网红主播合作以吸引消费者眼球, 二是需要降价以 刺激消费者购买, 成本上升, 利润压缩, 对普通商家 而言往往是 “赔本赚吆喝”。而网红主播、 $\mathrm{MCN}$ 机构 等却赚得盆满钵满 ${ }^{[42]}$ 。

\section{5. 实践启示}

在对直播带货的定义、类型、特点、作用机制以 及影响的系统梳理与分析基础上, 本文从主播、商家、 $\mathrm{MCN}$ 机构、政府以及直播平台这几个方面提出了实践 启示。

\section{1. 带货主播群体层面}

从上文的 SOR 理论和信息源理论可知, 主播的可 信性、吸引性、互动性、娱乐性和专业性等属性吸引 观众。匹配假说也表明自我与产品的匹配程度越高,
消费者购买意愿的可能性就越高。因此主播首先要找 准自己的定位，例如草根出身的网红可以直播售卖物 美价廉的大众产品, 明星带货选择价格相对较高的奢 侈品。实践中, 灵活识别顾客的沟通风格, 并调整自 身的沟通风格与其相匹配, 结合销售商品种类采取有 效的互动方式, 在互动中引领消费者积极交流, 做到 专业性与娱乐性并重, 以刺激消费者的情绪反应并增 强感知信任 ${ }^{[43]}$ 。最后, 电商直播要用创造性的思维为 受众塑造积极图景与想象, 引导积极向上的直播内 容。

\section{2. 商家层面}

首先, 企业不要盲目跟风迷信直播带货神话, 自 身产品和服务的质量才是重点, 直播只是种营销手 段, 不能本末颠倒, 应在权衡成本利润的基础上考虑 是否邀请网红主播直播带货。另外, 企业在选择网红 进行直播营销时, 要根据产品特点和目标客户来选择 与产品匹配的适合网红。对于规模并不大的小企业可 以采取商家自播的方式, 让员工甚至老板直播带货, 以节约成本。

\section{3. $M C N$ 机构层面}

直播带货这一领域目前同质化严重并且质量参 差不齐。 $\mathrm{MCN}$ 机构（打造网红的团队）应依据地域资 源和文化, 发展和挖掘自身特性, 打造有特色的带货 主播, 实现差异化发展。比如, 直播带货可与公益相 结合, MCN 机构根据经济欠发达地区的文化特点、地 域特点、农产品特点等, 打造具有本地特色的网红人 物或团队, 进行电商直播销售, 带动地域经济的发展 ${ }^{[39]}$ 。

\section{4. 政府和平台层面}

政府部门应尽快健全相关法律法规, 提高直播带 货的准入门槛, 以 “扶持发展, 创新监管” 为原则, 带动直播电商行业的纵深发展。一方面, 进一步出台 和完善扶持政策以优化发展环境, 如, 对直播带货需 要的场地、资金、人才培养等各项生产要素予以支持。 另一方面, 对于直播带货出现的商品造假、侵权行为 等乱象, 政府应当加强科学监管以促进整个行业健康 发展, 如, 明确各方参与者的权与责, 完善信用评估 和风险评估体系等 ${ }^{[11]}$ 。

各大电商平台或直播平台是行业规范化发展的 第一责任主体。净化网红直播环境, 平台也应该担负 起监管责任。例如，出台全面的 “直播间违规管理条 例” “主播自律公约”、及时处理消费者投诉等。

\section{6. 结论}

尽管近两年学者们在直播带货方面进行了许多 探索性研究, 但由于直播带货作为一个理论课题引入 营销领域的时间并不长, 现有文献对直播营销的研究 
尚存在一些不足, 有待进一步探讨。本文认为未来的 研究可以关注以下两个方面:

首先, 理论方面, 现有实证研究大多是从感知价 值理论、SOR 模型、信息源特性等理论探讨直播带货 如何影响消费者行为。未来可引入其他理论。例如, 运用刻板印象（Stereotype Content Mode1, SCM）, 研究热情与能力型主播对消费者购买意愿的影响。

其次, 参与对象方面, 直播带货包含电商主播、MCN 机构、商家、平台、消费者以及产品服务等多种要素, 现有研究大多从主播属性和直播特征的角度出发探 讨直播对消费者的影响, 未来可从其他参与要素展开 讨论。例如, 现在直播电商行业呈现内容精细化、带 货品类垂直化的发展趋势, 可把产品类型（实用品、 享乐品) 作为调节变量, 研究不同形象的网红在产品 类型的调节下对消费者购买意愿的影响。

\section{REFERENCES}

[1] Lu Z C, Xia H J, Heo S, et al.You Watch, You Give, and You Engage: a study of live streaming practices in China, In: 2018 ACM CHI Conference on Human Factors in Computing Systems, Montreal, QC, Canada, pp. 1-13.

[2] Meng Y B. Research on the regulation of the commercial publicity behavior of anchors in the live streaming commerce. J. People's Tribune, 2020 (25):

116-119.

[3] Iresearch. 2021 China Live streaming commerce Industry Research Report[EB/OL]. http://report.iresearch.cn/report/202109/3841.shtml

[4] Sun Y, Shao X, Li X, et al. How live streaming influences purchase intentions in social commerce: An IT affordance perspective. J. Electronic Commerce Research and Applications, 2019,37.

[5] Pei X L, Deng H M. The Process of Value Co-creation Behavior about Online Live Shopping in E-commerce Platform Based on the Taobao Live Shopping. J. Chinese Journal of Management, 2020,17(11):1632-1641.

[6] Wei H, Gao J S, Duan F F. The Impact of Information Interaction on User Engagement Behavior in Live Streaming Commerce. J. Information Science, 2021,39(04):148-156.

[7] Cai J, Wohn D Y, Mittal A, et al. Utilitarian and Hedonic Motivations for Live Streaming Shopping.J. Interactive Experiences for TV and Online Video, 2018.

[8] Park H J, Lin L M. The effects of match-ups on the consumer attitudes toward internet celebrities and their live streaming contents in the context of product endorsement. J. Journal of Retailing and Consumer Services, 2020,52.

[9] Meng L, Liu F J, Chen S Y, Duan S. Can I Evoke You? A Study on the Influence Mechanism of Information Source Characteristics of Different Types of Live Broadcasting Celebrity on Consumers' Willingness to Purchase. J. Nankai Business Review, 2020,23(01):

131-143.

[10] Li X R, Zhu S Y. Reason Analysis and Countermeasures of network live broadcast chaos.J. People's Tribune, 2020(19):64-65.

[11] Liu Y Z, Li X Q. Development characteristics and trends of China's live streaming commerce.J. Media, 2021(14):26-29.

[12] Han X Y. Trait Analysis and Governance Research of Network Live Broadcast Marketing_—From the

Perspective of Double Cycle Development Pattern. J. Henan Social Sciences, 2021,29(06):102-110.

[13] Apiradee W, Nuttapol A. The role of live streaming in building consumer trust and engagement with social commerce sellers. J. Journal of Business Research, 2020,117(prepublish).

[14] Liu Y, Li Q, Yin M.Research on the Influence of Webcast Shopping Features on Consumer Buying Behavior. J. Soft Science, 2020,34(06):108-114.

[15] Gu Y W. Research on the influence of e-commerce live broadcast on consumers'purchasing decision. J. Price:Theory \& Practice, 2020(02):124-127.

[16] Fan X J, Jiang X Y, Ni R R, et al. Influence of Interactivity of Mobile Live-Video Broadcaston Intention of Continuous Use of Users. J. Journal of Systems \& Management, 2020,29(02):294-307.

[17] China Netcasting Services Association. 2021 China Network Audio-Visual Development Research Report[EB/OL].

http://www.cnsa.cn/home/industry/industry_week.h tml.

[18] Yan X X, Dong Y H, Zhang M M, et al. Research on the Impact of Live Broadcasting on Consumers' Buying Behavior-Intermediate by perceived value. J. Price:Theory \& Practice, 2021(06):137-140.

[19] Kang K, Lu J, Guo L, et al. The dynamic effect of interactivity on customer engagement behavior through tie strength: Evidence from live streaming commerce platforms. J. International Journal of 
Information Management, 2020(prepublish).

[20] Zhao D W, Feng J X. The Impact of Characteristics of E-commerce Anchor as a Key Opinion Leader on Consumers' Purchase Intention. J. Commercial Research.,2021(04):1-9.

[21] Han $\quad X \quad Y, \quad X u \quad$ Z L.Impacts of E-Commerce Anchor Attributes on Co nsumers'Willingness to Buy Online: Research Base $\mathrm{d}$ on the Grounded Theory. J. Foreign Economics \& Management, 2020,42(10):62-75.

[22] Zhao B G, Wang Y F.The impact of the characteristics of e-commerce anchors on consumers' willingness to buy. J.Commercial Research , 2021(01):1-6.

[23] Xu H, Qu H J, Cai J Z. Influencing Factors of Apparel Consumers' Impulse Purchase Intention in the Context of Network Broadcast. J. Journal of Donghua University (Natural Science), 2020:1-11.

[24] Feng J, Lu M. The Empirical Research on Impulse Buying Intention of Live Marketing in Mobile Internet Era. J. Soft Science, 2020,34(12):128-133.

[25] Singh S, Singh N, Kalinić Z, et al. Assessing determinants influencing continued use of live streaming services: An extended perceived value theory of streaming addiction[J]. Expert Systems with Applications, 2021,168:114241.

[26] Ma H, Mei H. Empirical Research on the Decision-Making Influence Factors in Consumer Purchase Behavior of Webcasting Platform[M]//Cham: Springer International Publishing, 2018:1017-1028.

[27] Liu J, Zou Y J, Liu Z X. Analysis of the influence factors of consumers' purchase willingness in the live broadcast commerce based on the SEM model. J. Statistics \& Decision, 2021,37(07):94-97.

[28] Choi S M, Rifon N J. It Is a Match: The Impact of Congruence between Celebrity Image and Consumer Ideal Self on Endorsement Effectiveness. J. Psychology \& marketing, 2012,29(9):639-650.

[29] Yoo J, Jin Y J. Reverse Transfer Effect of Celebrity-Product Congruence on the Celebrity's Perceived Credibility. J. Journal of promotion management, 2015,21(6):666-684.

[30] Gong W, Li X. Engaging fans on microblog: the synthetic influence of parasocial interaction and source characteristics on celebrity endorsement. J. Psychology \& Marketing, 2017,34(7):720-732.

[31] Lee E M, Park S Y, Rapert M I, et al. Does perceived consumer fit matter in corporate social responsibility issues? J. Journal of Business Research, 2012,65(11):1558-1564.

[32] Sirgy M J, Lee D J, Johar J S, et al. Effect of self-congruity with sponsorship on brand loyalty.J. Journal of Business Research, 2008,61(10):1091-1097.

[33] Chung S, Cho H. Fostering Parasocial Relationships with Celebrities on Social Media: Implications for Celebrity Endorsement. J. Psychology \& Marketing, 2017,34(4):481-495.

[34] Li Q, Gao X Y, Xu X Y, et al. A Study on Viewers' Information Processiong and Purchase Intention in Live Streaming Commerce. J. Chinese Journal of Management, 2021,18(06):895-903.

[35] Li M, Hua Y. The Influence of Presence on Purchase Intention in Live Streaming Commerce_-from the Perspective of Vicarious Learning. J. China Business And Market, 2021,35(08):81-92.

[36] Guo R, Ye Y Q, Luo Y, et al. Which Kind of Stories Are More Helpful to Enhancing the National Brand Confidence in Webcast: Also on the Mediating Effect of Cultural Transportation and Regulating Effect of Time Anxiety. J. Journal of East China Normal University (Humanities and Social Sciences) , 2020,52(06):164-177.

[37] Fan X M, Wang X Y, Yang Y. The Impact of Network Spokesperson Characteristics on Consumer Brand Relationship Investment from the Consumer Perceived Perspective. J. Nankai Business Review, 2018,21(05):64-72.

[38] Zhang H, Dong Z Q, Wang H S.The Influence of Value Co-Creating on Fashion Design Attributes:Scale Development and Empirical Study. J. Chinese Journal of Management, 2017,14(09):1351-1361.

[39] Zan M Y, Wang Z B. E-commerce Live Streaming of Agricultural Products: a New Mode of Poverty Alleviation. J. Issues in Agricultural Economy, 2020(11):77-86.

[40] Chen J.Exploring the Path for Law-based Regulation of "Livestream Shopping". J. Frontiers, 2020(17):124-127.

[41] Wang B,Gao G W. Media Turn under the Catalytic Effect of the Pandemic: Motivation, Essence and Potential Risk of Live Streaming Shopping. J. Editorial

Friend, 2020(10):13-20.

[42] Yao L Q, Yu H X. The prosperity and chaos of live streaming shopping. J. People's Tribune, 2020(25):85-87. 
[43] Wu N, Ning C H, Gong X X.Research on the Influence Mechanism of Comm unication Style Similarity on Purchase Intention in Live Streaming Marketing. $\mathrm{J}$. Foreign Economics \& Management,2020,42(08):81 -95 . 